\title{
ARTICLE Design and fabrication of silicon-tessellated structures for monocentric imagers
}

\author{
Tao Wu, Stephen S. Hamann, Andrew C. Ceballos, Chu-En Chang, Olav Solgaard and Roger T. Howe
}

Compared with conventional planar optical image sensors, a curved focal plane array can simplify the lens design and improve the field of view. In this paper, we introduce the design and implementation of a segmented, hemispherical, CMOS-compatible silicon image plane for a 10-mm diameter spherical monocentric lens. To conform to the hemispherical focal plane of the lens, we use flexible gores that consist of arrays of spring-connected silicon hexagons. Mechanical functionality is demonstrated by assembling the $20-\mu \mathrm{m}$-thick silicon gores into a hemispherical test fixture. We have also fabricated and tested a photodiode array on a siliconon-insulator substrate for use with the curved imager. Optical testing shows that the fabricated photodiodes achieve good performance; the hemispherical imager enables a compact $160^{\circ}$ field of view camera with $>80 \%$ fill factor using a single spherical lens.

Keywords: curved image plane; monocentric imager; photodiode; recessed negative trench profile

Microsystems \& Nanoengineering (2016) 2, 16019; doi:10.1038/micronano.2016.19; Published online: 23 May 2016

\section{INTRODUCTION \\ Motivation}

Curved image planes that mitigate spherical aberration are essential for wide-angle, monocentric imaging technology to be generally useful ${ }^{1}$. Multiple efforts have been devoted toward developing monocentric imagers. Ford et al. $^{1-3}$ designed a monocentric triplet with a spherically polished fiber bundle that conforms to the lens and acts as a spherical-to-planar coupler to a CMOS imager. Crosstalk and the mode volume of the fibers constrain the pixel size and fill factor. These limitations motivate an alternative approach - a curved imaging plane. Sony has developed a curved CMOS imager by controlling bending stress ${ }^{4}$, which shows a $1.4 \times$ improvement in sensitivity and a $5 \times$ reduction of dark current; however, the radius of the curvature is too large for full hemispherical coverage of a miniature spherical lens. Rogers et al. ${ }^{5-8}$ demonstrated a hemispherical camera, consisting of isolated sensor clusters attached to an elastic material; however, the sensor area has a low fill factor, and the pixel density is nonuniform because of stretching. Dinyari et al. ${ }^{9,10}$ and Mathieson et al. ${ }^{11,12}$ reported a technique to develop curved monolithic silicon focal plane arrays for retinal implants; however, the process requires backside thinning, and metal interconnections have yet to be demonstrated.

In addition to the motivation to achieve a miniature monocentric imager, curved silicon structures can enable a variety of applications in flexible electronics and the Internet of Things ${ }^{13-15}$. Therefore, we seek to address the challenge of developing a lowcost, CMOS-compatible process for silicon-based flexible electronics by demonstrating a miniature hemispherical curved silicon substrate for monocentric imagers.

\section{Concept}

We seek to construct a flexible silicon surface that can conform to a hemispherical shell, by using planar lithographic techniques.
Although it is known that a perfect hemispherical shell cannot be constructed by folding a plane, a hemispherical surface can be approximately formed from planar structures by assembling flexible gore segments. The curve for a perfectly conformal gore segment is described by

$$
y= \pm r \tan (\pi / n) \sin (x / r)
$$

where $n$ is the number of gores ${ }^{16}$.

Figure 1 shows the design concept of a compact monocentric imager. Within each gore pattern governed by Equation (1), tessellated hexagonal structures, connected by spring bridges, form a flexible network. Each hexagonal cell can be up to several hundred microns in diameter. Using current CMOS active-pixel image sensor (APS) technologies with pixel sizes between 1 and $4 \mu \mathrm{m}^{17}$, thousands of pixels can be fabricated on one hexagonal cell, and hundreds of hexagonal cells can be accommodated in one gore. The bridges function as electrical connections and act as deformable springs that provide mechanical flexibility between semi-rigid hexagonal cells. The top-right sketch of Figure 1 indicates such an aberrationfree monocentric imager design with a hemispherical focal plane; the single ball lens can achieve a wide field of view with a significant reduction of the imager size. To demonstrate the manufacturability of this design, we have fabricated and tested photosensitive diodes on the hexagon cells and assembled the tessellated planar silicon into a hemispherical holder with a ball lens.

\section{MATERIALS AND METHODS}

\section{Structural design and simulation}

The use of thin substrates is essential to achieve hemispherically curved silicon surfaces because of the high Young's modulus and brittle fracture characteristic of silicon. Our fabrication process realizes thin substrates by employing silicon-on-insulator (SOI) 


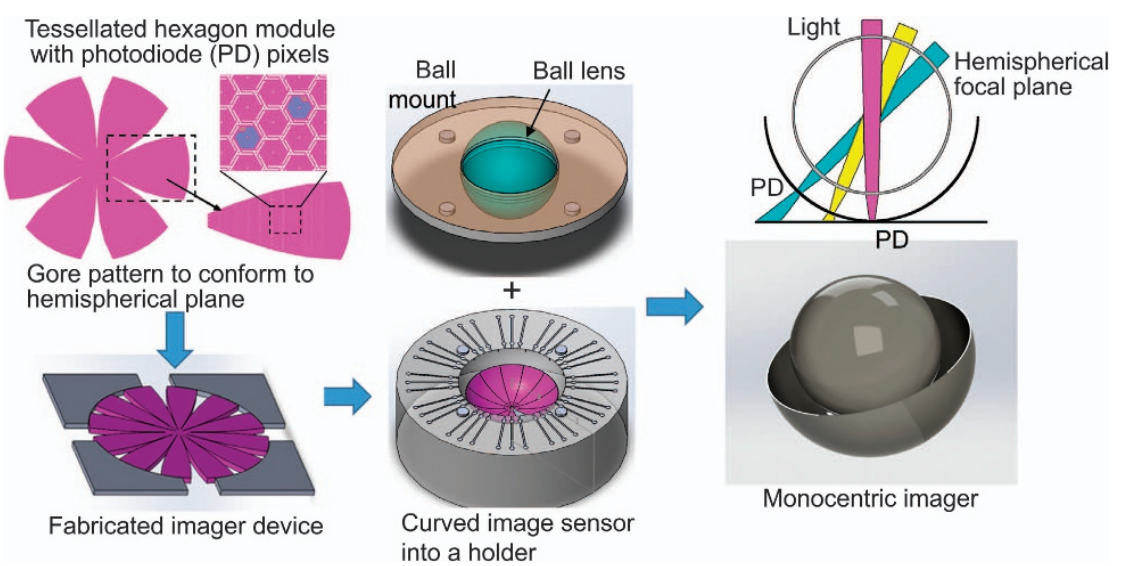

Figure 1 A design concept of a compact monocentric imager, which consists of a tessellated monocentric imager with a customized ball lens and a curved sensor holder. The top-right inset shows a monocentric imager with a hemispherical curved focal plane can simplify the lens design to just a single ball lens.

substrates, which are compatible with modern CMOS imager technologies ${ }^{18,19}$. The thickness of the device layer is subject to design tradeoffs. A thin device layer leads to better conformity and less stress, whereas a thick device layer provides mechanical strength, mitigates stress concentration and improves optical absorption. A ball lens with a diameter of $10 \mathrm{~mm}$ and a refractive index of 1.54 is used for our imager design. The corresponding focal length is $\sim 7.2 \mathrm{~mm}$. Each hexagonal unit cell can vary from tens of microns to hundreds of microns in diameter. The width of the interconnect bridges and trenches must be between 4 and $8 \mu \mathrm{m}$ to accommodate metal lines of width 2 to $4 \mu \mathrm{m}$. Because the hexagonal cell has six directions for interconnection, the design rule is one-half of the spacing and clearance of each layer in conventional designs. This limits the line/space critical dimension in our design and affects the fill factor of the overall image sensor. In one of our primary designs, the diameter of the hexagonal cell is $200 \mu \mathrm{m}$, and the line and trench width is $4 \mu \mathrm{m}$. The corresponding fill factor is $\sim 80 \%$, assuming that the whole hexagonal unit cell is photosensitive. Optimizing the hexagon cell size and interconnect spring line/space width with improved alignment accuracy can further improve the fill factor.

COMSOL Multiphysics ${ }^{\circledR}$ (COMSOL, Inc., Burlington, MA, USA) is utilized to analyze and optimize the stress/strain distribution in the suspension connecting the hexagonal structures. An evaluating test structure of a $20-\mu \mathrm{m}$ Si device with a complete hexagonal unit cell and six half-cells interconnected by springs represents the tessellated hexagonal structure. Figure $2 a$ shows the surface stress distribution when one side of the structure (half-cell surface in the $+y$ direction) is fixed and the other side of the structure has a prescribed displacement along the $z$-direction on the order of $2 \mu \mathrm{m}$. This corresponds to the situation in which the planar $\mathrm{Si}$ structure is conformed to a hemispherical surface with finite deformation along the $z$-direction on each hexagonal unit cell. The three-dimensional (3D) surface stress distribution plot indicates that stress concentrates on the spring bridges. The main photosensitive area-that is, the hexagonal unit-is mostly stress free. Therefore, the influence of curving stress on photodiodes or potential CMOS transistor circuitry is minimized.

Two enlarged insets in Figure $2 \mathrm{a}$ show cross-section twodimensional views of the springs' stress distribution in two different locations. A further analysis of the maximum strain versus different displacements along the $z$-direction is presented in Figure $2 \mathrm{~b}$. The plot indicates that the maximum strain value of the test structure is linearly proportional to the displacement along the $z$-direction at a given device thickness. The maximum stress/strain can be significantly reduced by using a thinner Si device layer. Figures $2 c$ and $d$ evaluate the strain distribution of the test structure with $2-\mu \mathrm{m}$ displacement along the $+z$ and $-y$ directions, respectively. Because our approach does not require stretching of the device to conform to the hemispherical surface, the actual displacement along the $y$ direction should be nearly zero. The maximum strain concentration shown in Figure $2 \mathrm{~d}$ indicates the robustness of the device design subject to external vibration and stretching. With our target 7.2-mm imager plane, the average displacement of each hexagonal unit cell is $<2 \mu \mathrm{m}$, which results in a maximum strain concentration on the bridge far $<1 \%$. Therefore, any device structure thickness $<30 \mu \mathrm{m}$ would be adequate for achieving the required flexibility, with minimal stress concerns.

We further investigate the impact of stress on the metal/ dielectric stack in CMOS processes. Although current CMOS backend processes can include 10 or more metal interconnect layers, a common APS technique typically contains three or four metal layers for signal processing and readout ${ }^{20}$. We investigate stress in the metal/dielectric stack by adding multiple $\mathrm{Al} / \mathrm{SiO}_{2}$ layers atop a $10-\mu \mathrm{m}$ Si layer, with each $\mathrm{Al} / \mathrm{SiO}_{2}$ layer consisting of $0.5 \mu \mathrm{m}$ of $\mathrm{Al}$ and $1.5 \mu \mathrm{m}$ of $\mathrm{SiO}_{2}$. As shown in Figure 3, a structure consisting of two half-hexagon cells and an interconnecting spring is simulated. Figure 3 a shows the $3 \mathrm{D}$ view of the stress distribution in a structure of $10 \mu \mathrm{m}$ of $\mathrm{Si}$ and a $10-\mu \mathrm{m} 5$-layer $\mathrm{Al} / \mathrm{SiO}_{2}$ stack, compared with that in the structure of $20 \mu \mathrm{m}$ Si in Figure 3c. Similar to the stress simulation in Figure 2a, the maximum stress is present along the sharp spring corner. Along the middle part of the spring, stress concentrates on the surface. Figure $3 \mathrm{~b}$ illustrates the cross section of the springs on four types of stack structures. It can be seen that stress concentrates along the silicon substrate surface, whereas the interfaces between the metal/dielectric stack have lower stress. This phenomenon results from the lower Young's moduli of $\mathrm{Al}$ and $\mathrm{SiO}_{2}$ compared with that of silicon. The total stack thickness also contributes to the maximum stress value. A summary of the maximum stress values of the four types of stack structures in Figure $3 \mathrm{~b}$ is provided in Table 1. The maximum stress increases with increasing total thickness of the structure, which is consistent with Figure $2 \mathrm{~b}$. The minimum thickness of the silicon is constrained by the required depletion depth in the photodiodes. The metal/dielectric layers contribute to the total thickness and increase the maximum stress and strain. Our design uses $20 \mu \mathrm{m}$ of $\mathrm{Si}$ with one metal/dielectric stack as a proof of concept for structural design and process compatibility. From Table 1, the maximum stresses in a $10 \mu \mathrm{m}$-thick silicon with a 
a

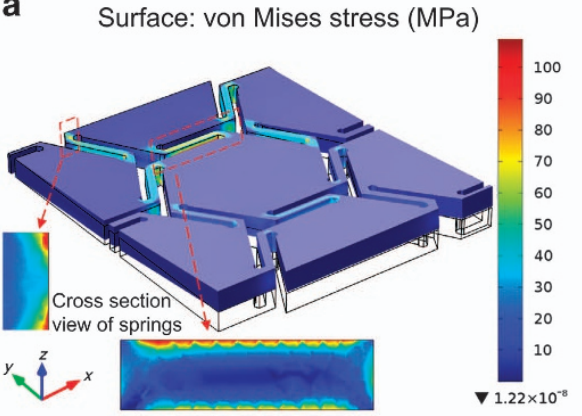

C b

Maximum strain in the testing structure

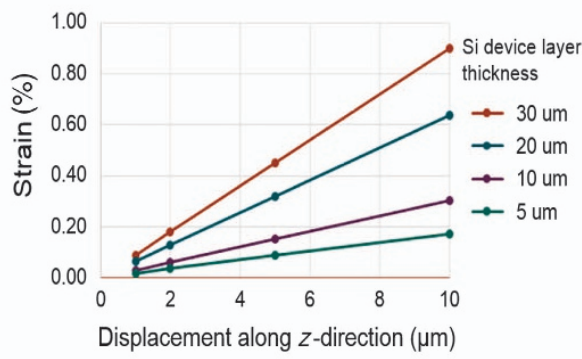

d

d Surface: First principal strain

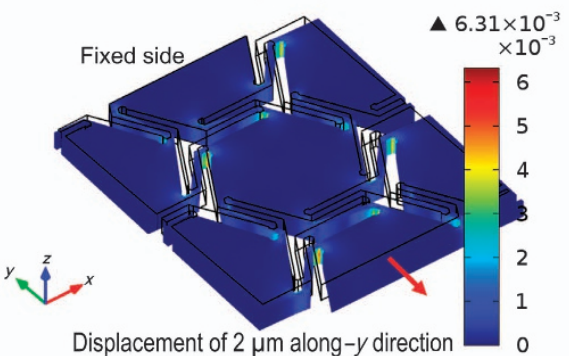

Figure 2 (a) Surface stress simulation of a test structure with one end fixed and the other end with a prescribed z-direction displacement of $2 \mu \mathrm{m}$; two insets show cross-section views of the interconnecting springs' stress distribution in two different locations; (b) the maximum strain concentration of the test structure as a function of $z$-direction displacement with different device layer silicon thicknesses; surface strain distribution with a prescribed displacement of $2 \mu \mathrm{m}$; (c) along the $+z$ direction; and (d) along the $-y$ direction.

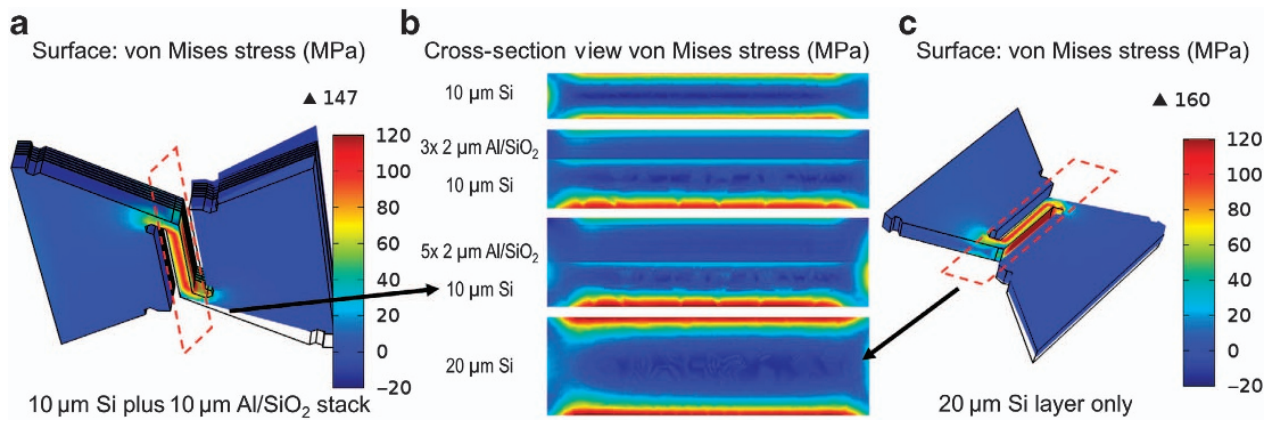

Figure 3 (a) Surface stress simulation of a test structure with a $10-\mu \mathrm{m} \mathrm{Si} \mathrm{layer} \mathrm{and} \mathrm{a} 5$-layer $\mathrm{Al} / \mathrm{SiO}_{2} \mathrm{metal} /$ dielectric stack. Each $\mathrm{Al} / \mathrm{SiO} \mathrm{O}_{2}$ layer consists of $0.5 \mu \mathrm{m}$ of $\mathrm{Al}$ and $1.5 \mu \mathrm{m}$ of $\mathrm{SiO}_{2}$. (b) Cross-section stress distribution of 4 different stacks: a 10- $\mu \mathrm{m} \mathrm{Si} \mathrm{layer,} \mathrm{a} \mathrm{10- \mu m} \mathrm{Si} \mathrm{layer} \mathrm{and}$ a 6- $\mu \mathrm{m} \mathrm{Al} / \mathrm{SiO}_{2}$ stack, a $10-\mu \mathrm{m} \mathrm{Si} \mathrm{layer} \mathrm{and} \mathrm{a} 10-\mu \mathrm{m} \mathrm{Al} / \mathrm{SiO}_{2}$ stack and a $20-\mu \mathrm{m} \mathrm{Si} \mathrm{layer.} \mathrm{(c)} \mathrm{Surface} \mathrm{stress} \mathrm{simulation} \mathrm{of} \mathrm{a} 20-\mu \mathrm{m} \mathrm{Si} \mathrm{test} \mathrm{structure.}$

Table 1 Maximum stress values in the spring

\begin{tabular}{lcccc}
\hline Stress value $(\mathrm{MPa})$ & $10-\mu \mathrm{m}$ Si layer & $10-\mu \mathrm{m} \mathrm{Si}, 3 \times 2 \mu \mathrm{m} \mathrm{Al} / \mathrm{SiO}_{2}$ stack & $10-\mu \mathrm{m} \mathrm{Si}, 5 \times 2-\mu \mathrm{m} \mathrm{Al} / \mathrm{SiO}_{2}$ stack & $20-\mu \mathrm{m} \mathrm{Si} \mathrm{layer}$ \\
\hline Max stress inside the spring & 117 & 138 & 147 & 160 \\
Max stress in the cross section view of the spring & 95.8 & 99.4 & 109 & 121 \\
\hline
\end{tabular}

$10 \mu \mathrm{m}$-thick, 5-layer metal/dielectric stack, typical of CMOS imagers, are similar to those of our proof of concept design.

\section{Photodiode design and simulation}

To demonstrate that devices made with a CMOS-compatible process could function properly and maintain performance with the flexure of the substrate, we fabricated and tested basic photodiodes on an SOI substrate. The process employs a modified $p$ substrate and $n$ well design. Pinned photodiodes, which are used in commercial CMOS imagers ${ }^{21}$, are compatible with this process and can be included in future device implementation. Various diode layouts were evaluated using the Synopsys ${ }^{\mathbb{R}}$ Sentaurus TCAD simulator (Synopsys Inc., Mountain View, CA, USA) 
a

Photodiode layout and doping schematic

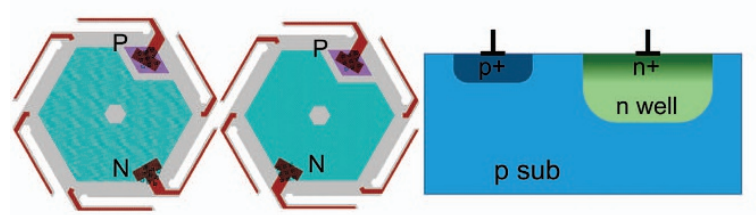

C

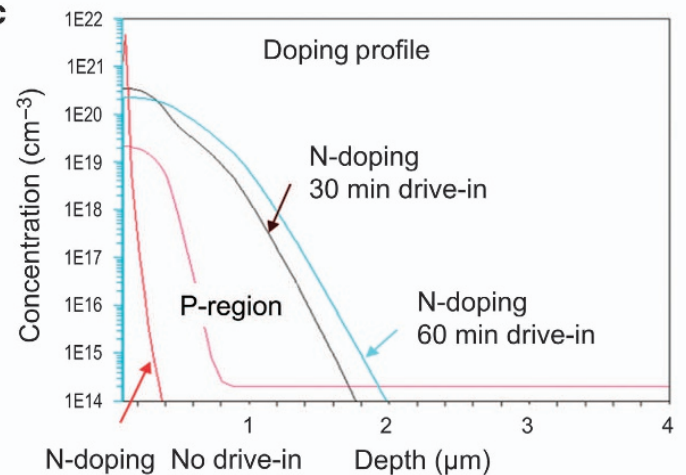

b

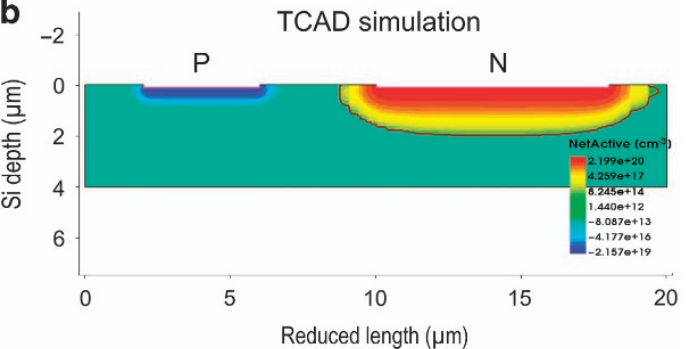

d

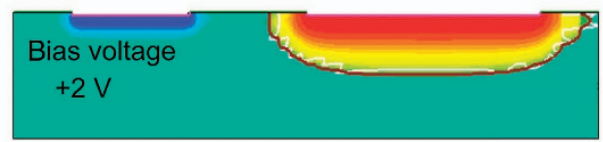

e

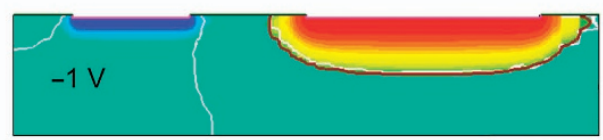

f

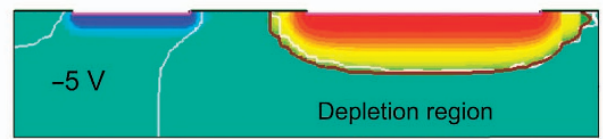

Figure 4 Sentaurus simulation on the photodiode (a) layout; (b) cross-section doping profile; (c) doping profile on the $p+$ and the $n+$ regions after ion implantation and different drive-in conditions; cross-section doping profile and depletion boundary (white line) at a bias voltage of (d) $+2 \mathrm{~V},(\mathbf{e})-1 \mathrm{~V}$, and (f) $-5 \mathrm{~V}$.

to optimize the process design. Figure 4a shows two photodiode layouts and a schematic cross-section doping profile. Figure $4 \mathrm{~b}$ shows the TCAD simulation results on the depletion region. The dimensions used in the simulation are scaled except for the PN junction spacing to reduce computation time. Figure $4 \mathrm{c}$ shows the doping profile of the $p$ and $n$ regions. The simulated junction depth after $60 \mathrm{~min}$ of annealing is $\sim 1.9 \mu \mathrm{m}$. The $n+$ region is formed in a gas-phase doping furnace using $\mathrm{POCL}_{3}$ as a phosphorus source, and the surface phosphorus concentration is as high as $1 \times 10^{20} \mathrm{~cm}^{-3}$ to make ohmic contact with the subsequent metal layer. A heavily doped $\mathrm{p}+$ region is formed by boron ion implantation at $20 \mathrm{keV}$ with a dose of $5 \times 10^{15} \mathrm{~cm}^{-2}$. A surface boron concentration of $2 \times 10^{19} \mathrm{~cm}^{-3}$ is achieved. Figures $4 \mathrm{~d}-\mathrm{f}$ show the simulated depletion region of the $\mathrm{PN}$ junction with a bias of $+2,-1$, and $-5 \mathrm{~V}$, respectively. As the reverse bias voltage increases, the depletion region widens and moves closer to the $p+$ region. The junction depth extends several microns deep at $-5 \mathrm{~V}$, yielding sufficient photoabsorption for the proof of concept device.

\section{Photodiode addressing}

Because of its unique hexagonal geometry, conventional rowcolumn addressing is inappropriate for this imager. Inspired by the flower-shaped gore patterns described by Equation (1), we propose a polar coordinate system to address the individual hexagonal unit cells of the curved imager. As shown in Figure 5a, for a fully covered tessellated hexagonal network with a hexagonal unit cell of edge radius $r$, the hexagon center is the origin and is defined as layer 0 , a discrete radius $\rho$ represents different layers along the polar axis, and the angular position $\theta$ is determined by the numbering of the cell times the cell angle at each layer, as listed in Table 2 . Therefore, the radius $\rho$ and angular angle $\theta$ coordination for any hexagon cell at layer $n$ can be expressed at $(2 n r, 2 \pi m / 6 n)$, where $m$ is an integer $<6 n$. a

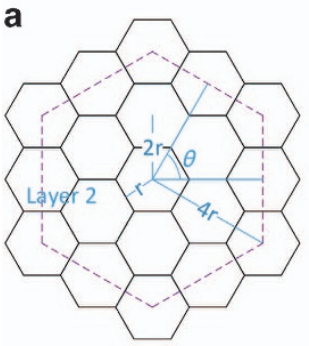

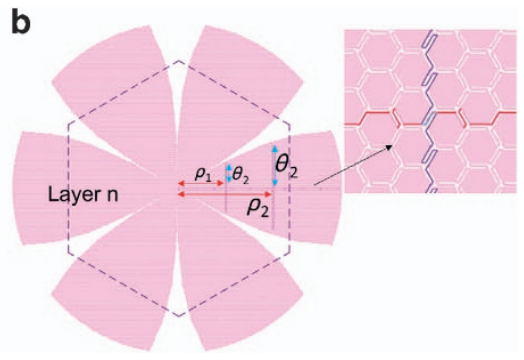

b
Figure 5 (a) The tessellated hexagon network with layered addressing concept; (b) a six-gore device design using a polar-like coordinate system $(\rho, \theta)$ to address individual hexagonal cells; the inset shows enlarged two-layer metal routing along the interconnect sprints.

Table 2 Summary of radius and cell angle for each tessellated hexagon network layer

\begin{tabular}{lccc}
\hline Layer & Radius $\rho$ & Number of cells & Cell angle $\theta$ \\
\hline Layer 0 & 0 & 1 & NA \\
Layer 1 & $2 r$ & 6 & $2 \pi / 6$ \\
Layer 2 & $4 r$ & 12 & $2 \pi / 12$ \\
Layer $n$ & $2 n r$ & $6 n$ & $2 \pi / 6 n$ \\
\hline
\end{tabular}

Two additional factors remain. First, as mentioned in the previous section, we fabricated one photodiode on each single hexagonal unit cell as a proof of concept, and the diameter of each hexagonal unit cell ranges from 100 to $200 \mu \mathrm{m}$, which is chosen by balancing the fill factor and release etching time. However, the pixel sizes of modern CMOS imagers have decreased 
a

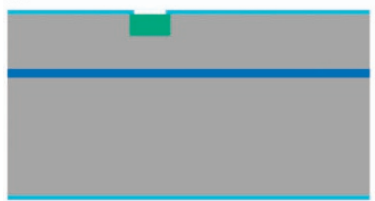

C

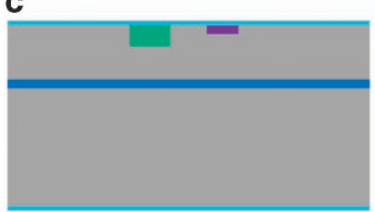

e

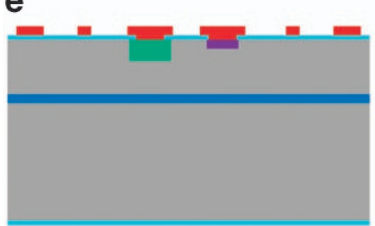

g

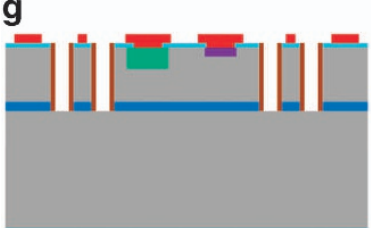

b

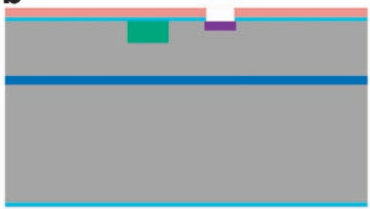

d

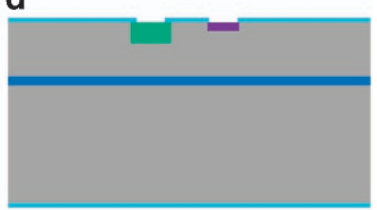

f

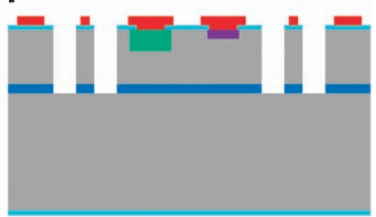

h
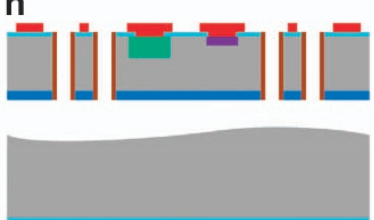

Si $\square$ Buried oxide $\square \mathrm{n}+$ diffusion area $\square \mathrm{p}+$ ion implantation area PR Surface $\mathrm{SiO}_{2} \square$ Sidewall Passivation Metal interconnect

Figure 6 Fabrication process flow: (a) form $\mathrm{n}+$ diffusion area; (b) form p+ ion implantation area; (c) grow new surface oxide; (d) pattern via for photodiode contacts; (e) deposit metal contact and interconnect; (f) pattern tessellated structures thru Si device and buried oxide layers; (g) sidewall passivation; (h) release the device by $\mathrm{XeF}_{2}$ etching. to $\sim 1 \mu \mathrm{m}$ or less, so each hexagonal unit cell could contain thousands of modern pixels, arranged in a continuous, planar silicon substrate. Thus, each hexagon cell can be treated as a group of thousands of pixels laid out on a row-column grid.

Second, the gore pattern has its own boundary governed by Equation (1). As the radius increases, the number of hexagonal cells will decrease to less than the full-coverage quantity $6 n$. The tessellated network is divided into several sections according to the numbers of gore patterns. Thus, each gore pattern could require one signal bus along the radial axis to address different layers.

Figure 5b shows the polar addressing coordinate for the hexagonal cells. In the first gore pattern, a bus line connects the origin and the outer edge through the interconnected spring hinges and hexagonal cell edge. The distance is defined by the polar length $\rho$. At different polar positions, another signal line is extended into each individual cell with a unique angular coordinate $\theta$. Only two metal layers are needed to connect all hexagonal cells.

There are several strategies to achieve seamless coverage on a spherical surface. One approach is to design gore patterns governed by Equation (1) that perfectly align each other and conform to a hemispherical surface. This approach requires very precise assembly. Another approach is to use two patterns of $n / 2$ center-connected segments that are slightly wider than Equation (1), and two patterns can overlap below on one side and above on the other. The addressing bus lines and readout circuitry could be laid out in this area without sacrificing usable photosensitive area.

\section{Fabrication}

The fabrication process starts on SOI wafers with a 20- $\mathrm{mm}$-thick device layer and a $0.5-\mu \mathrm{m}$-thick buried oxide (BOX). The device layer resistivity is $50-100 \Omega \mathrm{cm}$. The process flow is shown in Figure 6 (see Supplementary Information for process equipment details and recipe conditions). First, $100-\mathrm{nm}$ of thermal $\mathrm{SiO}_{2}$ is grown and patterned as a mask for $\mathrm{n}+$ doping. A phosphorus predeposition process is performed with a Tylan POCl3-doping

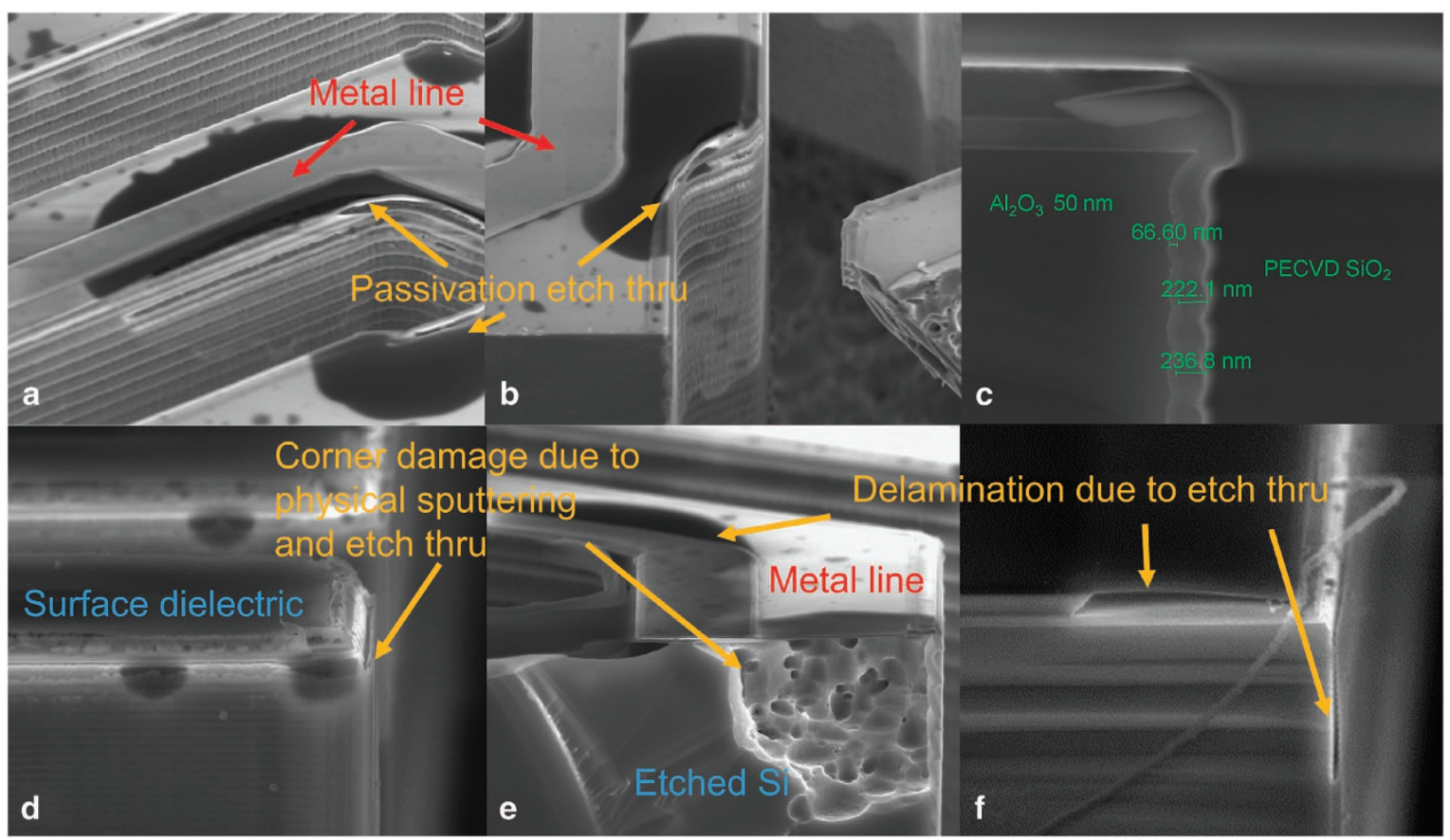

Figure 7 SEM images of devices showing (a and $\mathbf{b}$ ) surface dielectric and sidewall passivation delamination; (c) ultrasmooth sidewall passivation with 50-nm ALD $\mathrm{SiO}_{2}$ and 250-nm PECVD $\mathrm{SiO}_{2} ;(\mathbf{d}-\mathbf{f})$ trench corner damage due to enhanced etching. 
furnace followed by an annealing step at $1000^{\circ} \mathrm{C}$ for $30 \mathrm{~min}$, resulting in a sheet resistance of $\sim 5 \Omega \mathrm{sq}^{-1}$ on the $n+$ phosphorus doping region (Figure 6a). This forms a PN junction and also provides a good contact for the $n+$ region to the metal layer. Another thermal oxidation step is performed and patterned to define the opening for $p+$ boron implantation (Figure $6 b$ ). The ion implantation is conducted using $\mathrm{B}^{11}$ singly charged boron at $20 \mathrm{kV}$ at a dose of $5 \times 10^{15} \mathrm{~cm}^{-2}$. After ion implantation, a new $100-\mathrm{nm}$ surface thermal oxide is grown (Figure 6c), followed by a 20-min drive-in step at $1000^{\circ} \mathrm{C}$, resulting in a sheet resistance of approximately $5 \Omega \mathrm{sq}^{-1}$ on the $\mathrm{p}+$ phosphorus-doping region. Vias are then opened by etching through surface oxide layers from the contact regions (Figure $6 \mathrm{~d}$ ). Following that, a metal layer of $10-\mathrm{nm} \mathrm{Ti} / 100-\mathrm{nm} \mathrm{Pt}$ is deposited and patterned by a liftoff process to form the interconnect (Figure 6e).

After the photodiode circuitry has been fabricated, $\sim 250-\mathrm{nm}$ of PECVD $\mathrm{SiO}_{2}$ and 50-nm of $\mathrm{ALD} \mathrm{Al}_{2} \mathrm{O}_{3}$ are deposited atop the device as a surface dielectric stack and etch buffer layer. The tessellated hexagonal structure is patterned using a $3-\mu \mathrm{m}$ photoresist as a mask followed by reactive ion etching (RIE) on surface $\mathrm{Al}_{2} \mathrm{O}_{3}$ and $\mathrm{SiO}_{2}$ stack layers to expose the device silicon layer. The silicon layer is etched by deep RIE (DRIE) followed by another RIE etching of the $0.5-\mu \mathrm{m}$ barrier oxide layer (Figure $6 \mathrm{f}$ ). CMOS imagers include a thick metal-dielectric stack, which will require switching plasma etch chemistries during the hexagonal structure etch ${ }^{22}$. The thickness of the surface oxide etch buffer layer can be increased to allow for deeper etch depth. Alternatively, a temporary metal hard mask can be added.

Following the structure definition, a conformal coating of a pinhole-free, 50-nm ALD $\mathrm{Al}_{2} \mathrm{O}_{3}$ layer, and a 250-nm PECVD $\mathrm{SiO}_{2}$ or low-temperature oxide (LTO) layer are deposited as sidewall passivation and protection layers for the release etch. A directional $\mathrm{RIE}$ etch step is performed to remove $\mathrm{SiO}_{2}$ and then $\mathrm{Al}_{2} \mathrm{O}_{3}$, exposing the silicon handle substrate at the bottom of the trenches and giving access to the $\mathrm{XeF}_{2}$ release etch (Figure $6 \mathrm{~g}$ ). The structure is released by $\mathrm{XeF}_{2}$ dry etching (Figure $6 \mathrm{~h}$ ). Finally, a conformal coating of $5-10 \mu \mathrm{m}$ Parylene-C film is deposited to improve the mechanical robustness of the gores, allowing them to be transferred and mounted into a hemispherical device holder.

A high quality and CMOS-compatible sidewall passivation process is critical for the device release step. Our process uses ALD $\mathrm{Al}_{2} \mathrm{O}_{3}$ and PECVD $\mathrm{SiO}_{2}$ or LTO conformal films. ALD $\mathrm{Al}_{2} \mathrm{O}_{3}$ has better adhesion to silicon surfaces than other ALD films, but the ALD layer thickness is limited because of its very low deposition rate. A second low-stress layer of PECVD $\mathrm{SiO}_{2}$ or LTO is used to improve the sidewall passivation integrity and provide protection for the $\mathrm{ALD} \mathrm{Al}_{2} \mathrm{O}_{3}$ etch. The etching species for $\mathrm{Al}_{2} \mathrm{O}_{3}$ are $\mathrm{Cl}_{2}$ and
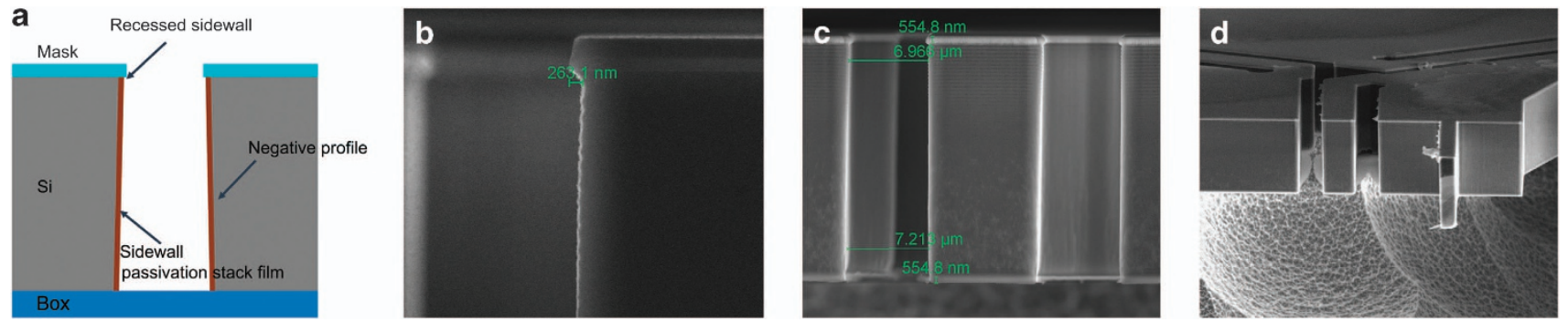

Figure 8 (a) The concept of recessed negative trench profile to preserve spacer film and quality; (b) an SEM image shows a recessed sidewall profile before passivation coating; (c) an SEM image of patterned SOI device layer with a recessed negative profile and passivation coating; (d) an SEM image of a fully released structure after $\mathrm{XeF}_{2}$ etching.
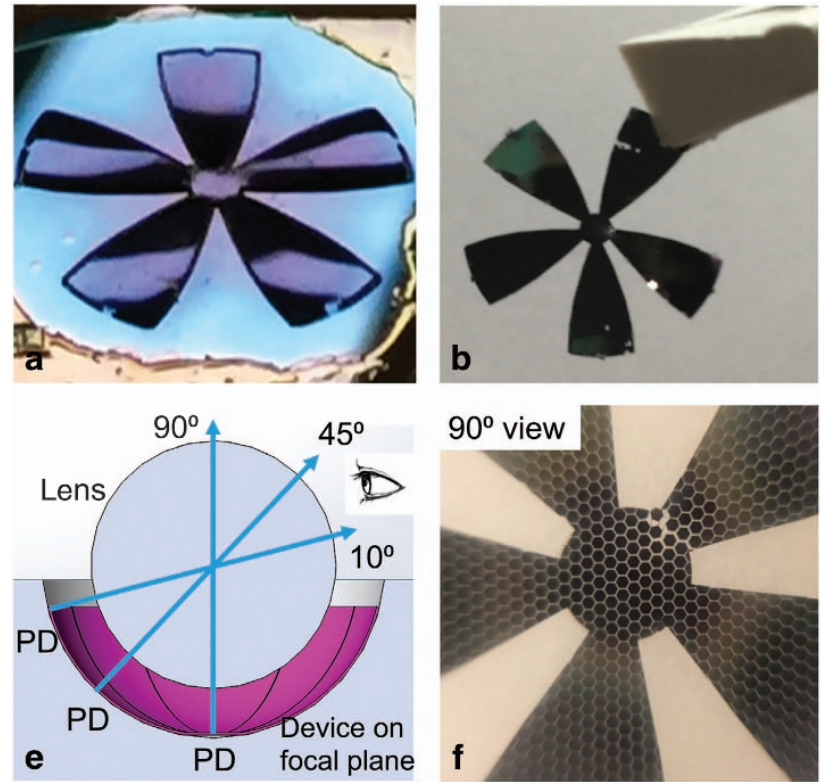
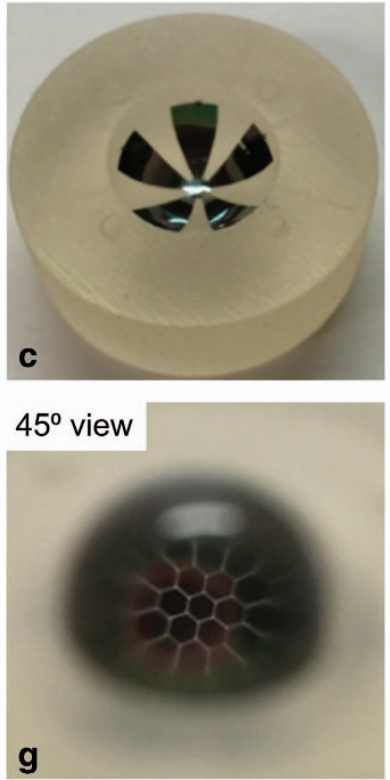
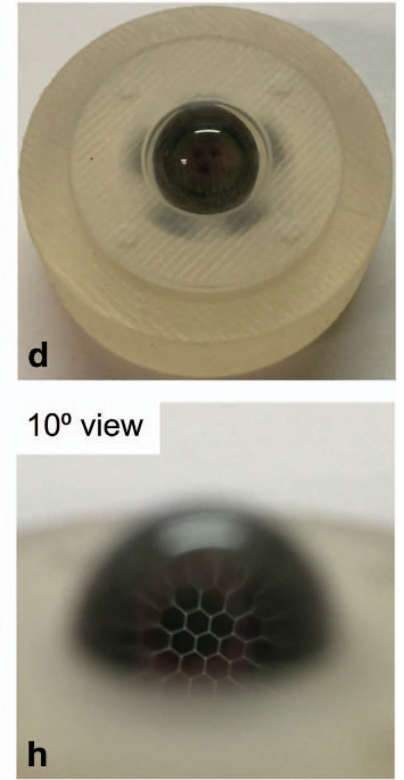

Figure 9 (a) A released five-gore structure, still attached to the silicon substrate with tethers; (b) a fully released five-gore structure before mounting into the fixture; (c) a released and curved device transferred into a hemispherical fixture; (d) a mounted monocentric imager; (e) a sketch illustrating the ray tracing approach by viewing through a ball lens into released structure; and images taken by a commercial camera through the ball lens at an angle of (f) $90^{\circ}(\mathbf{g}) 45^{\circ}$ (h) $10^{\circ}$ with respect to the horizon. 
$\mathrm{BCl}_{3}$, which etch many other materials. Significant delamination was observed initially because of poor sidewall passivation, as shown in Figures 7a and $b$. This problem was attributed to a relatively large scallop depth arising from the standard DRIE etch process. To mitigate delamination, we improved the silicon sidewall smoothness. A post-etch hydrogen annealing process requires very high-temperature annealing, much too high for CMOS compatibility. Therefore, we optimized the sidewall profile by utilizing the fast switching feature in a PlasmaTherm Versaline DSE silicon DRIE etcher. Figure 7c shows that a sub-100-nm scallop depth can be achieved with a spacer stack of 50-nm of ALD $\mathrm{Al}_{2} \mathrm{O}_{3}$ and 250-nm of PECVD $\mathrm{SiO}_{2}$.

However, even with a smooth trench sidewall profile, a number of defects are seen around the trench corners because of poor dielectric coverage over the metal lines and an enhanced etch rate on sharp edges, as indicated in Figures $7 d-f$. However, gas transport to the bottom of the trench slows the etch rate and results in incomplete etching. To overcome these challenges, we designed a recessed negative trench profile that successfully protected the ALD $\mathrm{Al}_{2} \mathrm{O}_{3}$ and PECVD $\mathrm{SiO}_{2}$ or LTO films near the top corner.

Figure $8 \mathrm{a}$ shows the sketch of a recessed negative sidewall profile. The sidewall passivation stack films, ALD $\mathrm{Al}_{2} \mathrm{O}_{3}$ and PECVD $\mathrm{SiO}_{2}$ or LTO, are coated underneath the mask with a recession of 200-300-nm, which matches or is slightly larger than the stackfilm thickness. This approach protects the passivation stack films from the physical sputtering near the corner edge. This sidewall recession depth can be tuned by timed etching with $\mathrm{CF}_{4}$-enriched gases after DRIE patterning. Figure $8 \mathrm{~b}$ shows a sidewall recess of $\sim 260-n m$ achieved using $\mathrm{SiO}_{2}$ as the top etching mask. Owing to the difficulty of achieving a perfect vertical sidewall profile, a slightly negative profile is achieved by linearly increasing the etching cycle time of DRIE in the PlasmaTherm DSE tool. Figure $8 \mathrm{c}$ shows the zoomed-out image of the trench profile with an $\sim 2^{\circ}$ negative sidewall, which has a negligible impact on the structural strength and functionality. Figure $8 \mathrm{~d}$ shows a cross-section image of the released structures after $\mathrm{XeF}_{2}$ etching.
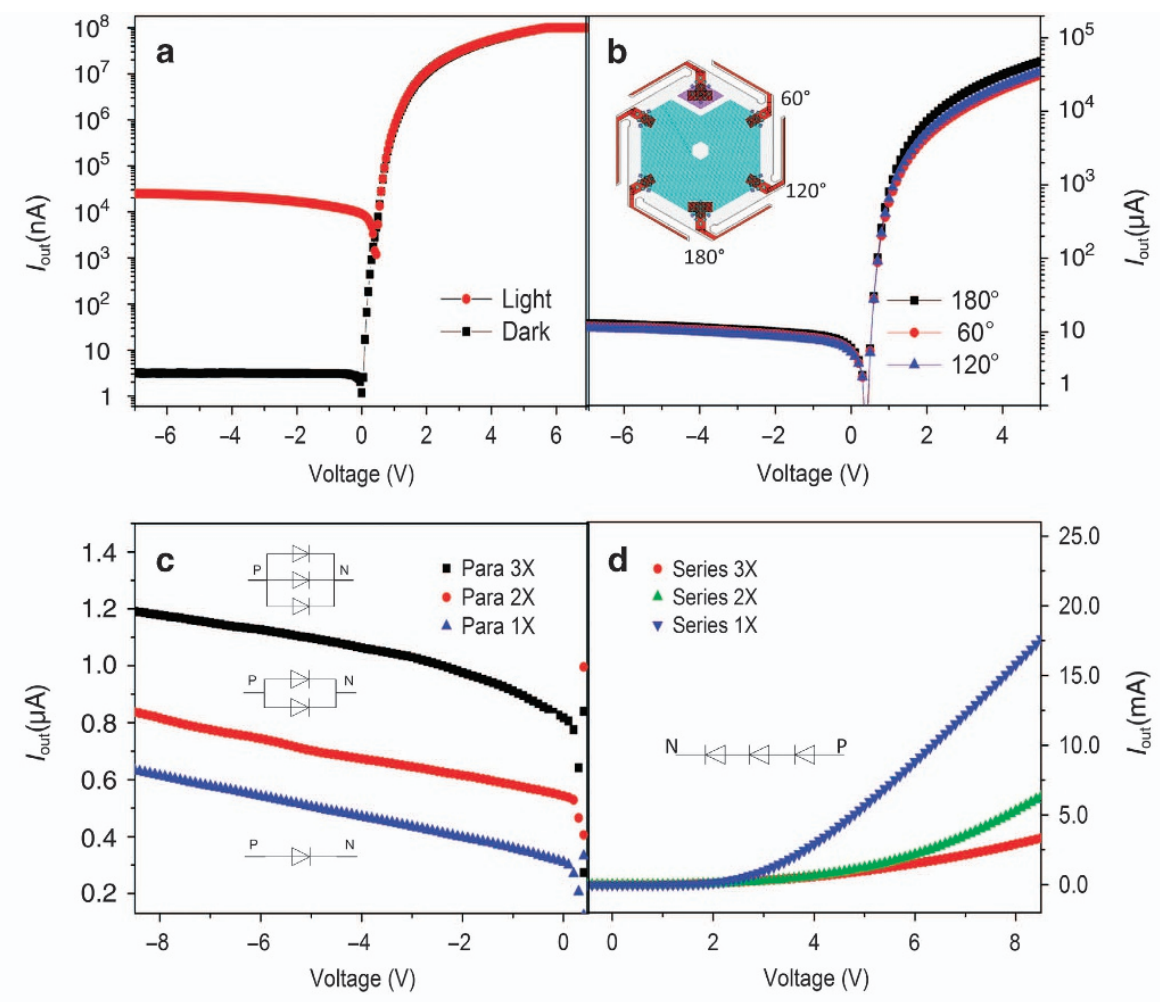

Pareto chart of measured dark current and On/Off current ratio distribution

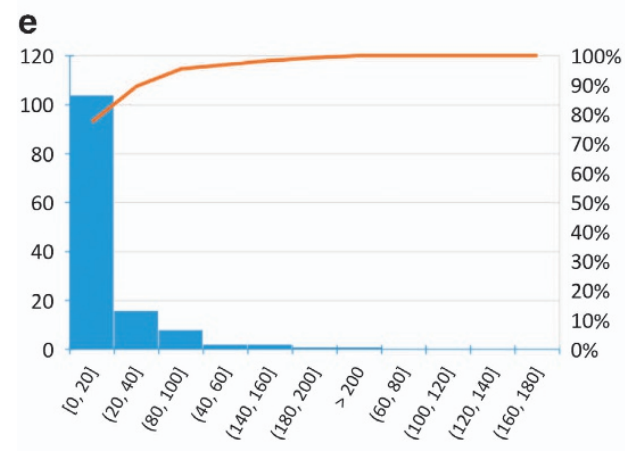

Dark current range $(\mathrm{nA})$

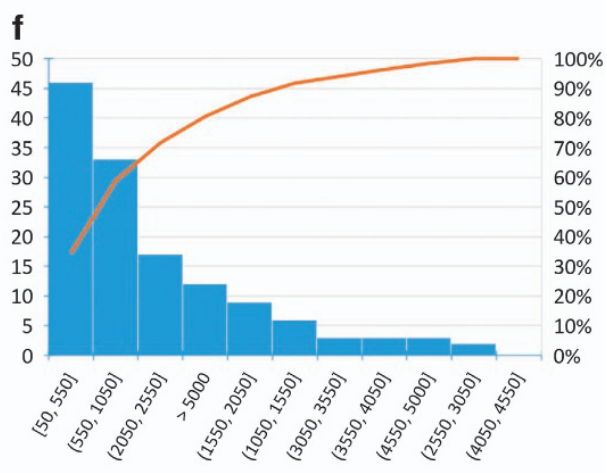

On/Off current ratio

Figure $10 \quad I-V$ characteristics of (a) one photodiode under microscope light and background dark environment, and (b) one photodiode on current under microscope light probed at a fixed p-region contact and different n-region contacts; $I-V$ characteristics of one, two, and three photodiodes in (c) parallel connection and (d) series connection; Pareto chart of measured (e) dark current and (f) on/off ratio distribution. 


\section{RESULTS AND DISCUSSION}

The released tessellated structure has tether supports connected to the substrate, as shown in Figure 9a. By carefully breaking those tethers, the $20-\mu \mathrm{m}$-thick silicon device with tessellated hexagonal structures is transferred and mounted in a 3D printed plastic test fixture, as shown in Figures $9 \mathrm{~b}-\mathrm{d}$. The fixture is manufactured using a high-resolution 3D printer (ProJet ${ }^{\circledR} 3500$ HD, 3D Systems Corporation, Rock Hill, SC, USA). Instead of reconstructing a photodetector mapping, the monocentric imager concept and performance can be preliminarily evaluated through a simple raytracing approach: the hexagonal structures on the hemispherical surface are imaged through the ball lens, as shown in Figure 9e. Figures $9 f-h$ are images taken by a commercial camera at viewing angles of approximately $90^{\circ}, 45^{\circ}$, and $10^{\circ}$, respectively. All three images show clearly visible hexagonal structures, verifying that the released gore is located on the hemispherical image plane. Using this technique, we verified that the field of view is $\sim 160^{\circ}$. Notably, the focal length can be adjusted by choosing a different ball lens material and refractive index.

Figure $9 f$ also indicates that fracture could occur during the transfer and assembly process, especially at the corner edges near the gore patterns. To minimize the possibility of damage during the assembly of released gores, the fixture's hemispherical surface is designed with a slightly smaller curvature. A half-hemispherical coverage is more preferable, in which two half-coverage sets of gores overlap each other to achieve full hemispherical coverage.

The optical performance of the fabricated photodiodes is characterized by measuring the current-voltage $(I-V)$ characteristics in the dark and under illumination. Most of our measurements were taken using illumination under a microscope and were performed before the release etch. Selected photodiodes were wire bonded for characterization after release. Figure 10a shows a typical characteristic of one photodiode in the dark and with microscope illumination. The dark current without background light is approximately $2-3 \mathrm{nA}$. A reverse bias voltage as high as $20 \mathrm{~V}$ is observed without breakdown. With only moderate microscope light illumination, the measured photocurrent is approximately 20-30 $\mu \mathrm{A}$, resulting in an on/off ratio of approximately 1000:1.

Figure $10 \mathrm{~b}$ shows $I-V$ curves of one photodiode from different n-region contacts. The $I-V$ curves are similar to each other, indicating that the surface potential is uniform across the n-doped diffusion area. This provides the flexibility to access the $n$-side contact from any of the hexagon cell springs that could be used for the routing network. Figures $10 \mathrm{c}$ and $\mathrm{d}$ plot the $I-V$ curves for up to three diodes in parallel and series, respectively. The parallel configuration (Figure 10c) clearly shows that the photocurrent at a negative bias voltage is tripled or doubled at $3 x$ or $2 x$ parallel connection, respectively. The series configuration (Figure 10d) indicates that the series resistance increases with multiple diodes connected in series and increasing diode operating forward voltage. These $I-V$ characteristics show that good photodiode circuitry can be fabricated in our CMOS-compatible process and conformed to hemispherical surfaces for monocentric imager applications.

Figures $10 \mathrm{e}$ and $\mathrm{f}$ show the distribution of measured photodiodes before release. The majority of tested devices show a dark
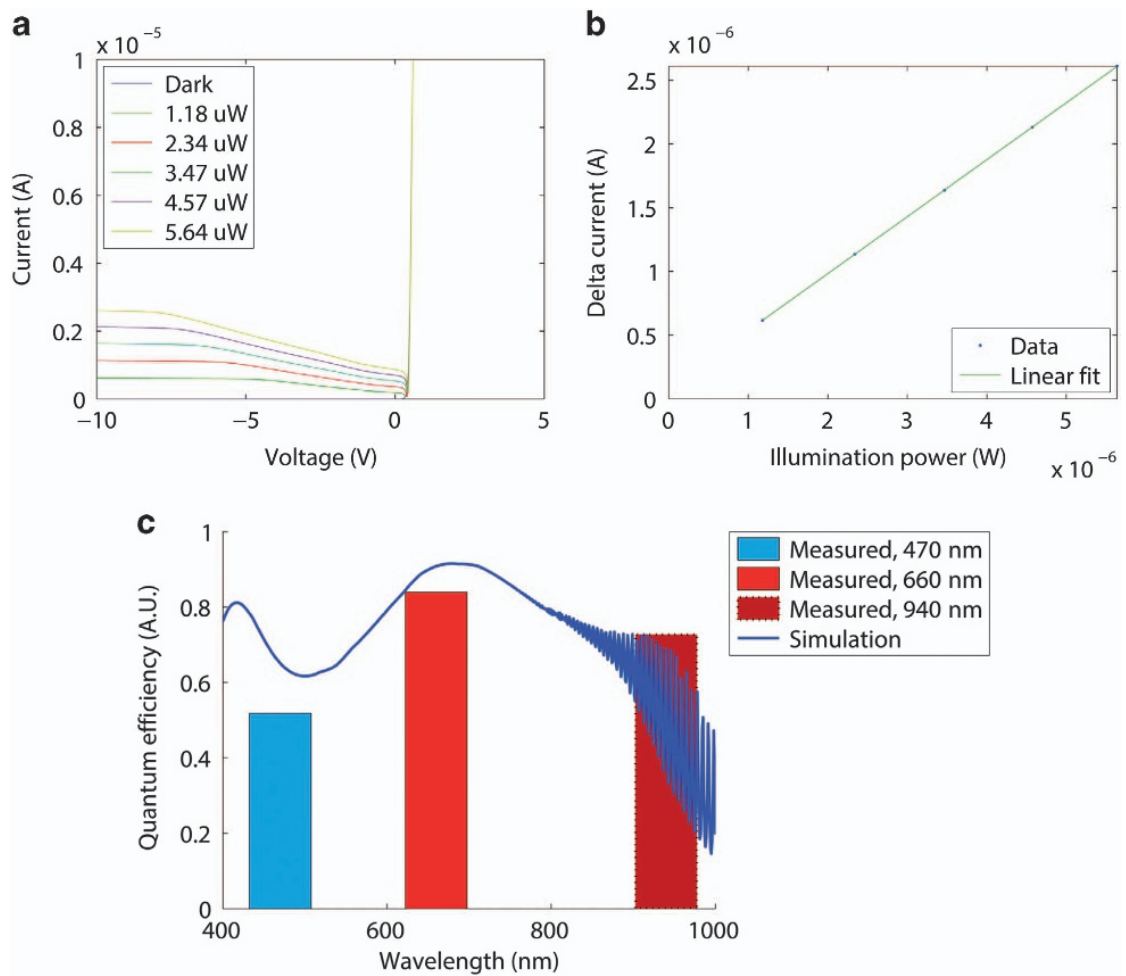

Figure 11 (a) I-V characteristics of a single hexagon cell under 660-nm LED light. The light source is a Thorlabs M660F1. The optical power, at the end of the microscope objective, is measured by a Newport 818-ST2 photodiode with a Newport 1835-C power meter. The ambient light is below $0.1 \mathrm{nW} \mathrm{cm} \mathrm{cm}^{-2}$. The displayed power readings have been adjusted to account for the size difference between the photosensitive area of the device and the spot size of the beam; (b) Current at $-10 \mathrm{~V}$ versus incident power, with dark current removed. The response is linear with a correlation coefficient $>0.99999$, suggesting linear minority carrier generation and collection; (c) Measured quantum efficiencies calculated from measured responsivities, compared with simulated results from a 1D transfer matrix method. The simulated structure is, from top to bottom, 350-nm of $\mathrm{SiO}_{2}, 20-\mu \mathrm{m}$ of Si, 500-nm of $\mathrm{SiO}_{2}$, and 550- $\mu \mathrm{m}$ of Si. The structure corresponds to the nominal layer thicknesses described in the fabrication section. Published optical constants ${ }^{23}$ are used in the calculation. Light sources used in the measurement are Thorlabs M470F1, M660F1, and M940F1. 1D, one-dimensional. 
current $<20 \mathrm{nA}$, whereas the on/off ratio has a larger range, with the majority falling between 100 and 1000 . This discrepancy could be due to several factors, such as process deviation, sidewall passivation uniformity and metal contact resistance variations.

Figures $11 \mathrm{a}$ and $\mathrm{b}$ show a set of $I-V$ measurements under calibrated illumination, demonstrating the linearity of the photodiode. A 660-nm light-emitting diode (LED) is coupled through a multimode fiber and a microscope objective to the device. The total incident power is estimated by scaling the measured LED power with the ratio of the device's photosensitive area to the test beam's spot size, assuming a uniform intensity profile. The gradual saturation of the reverse current is characteristic of the photodiode's geometry. As depicted in Figure 4, the depletion region expands vertically and improves photoelectron collection with increasing reverse bias. As shown in Figure $11 \mathrm{~b}$, with sufficient reverse bias, saturated reverse current is linear to incident illumination. The saturation behavior can be optimized by introducing modern pinned photodiodes and substrate doping engineering into our process. The slope in Figure $11 \mathrm{~b}$-namely, the responsivity-allows the quantum efficiency to be estimated. Figure $11 \mathrm{c}$ compares the quantum efficiencies at 470, 660, and 940-nm, each measured with a different light source, with the simulation results from a one-dimensional transfer matrix method implemented in-house. The simulated curve shows interference effects that are characteristic of our basic stack structure. The measured quantum efficiencies approximately match the simulation, indicating potential for further optical optimization.

\section{CONCLUSION}

We have demonstrated a fabrication process for a tessellated gore structure with interconnected hexagonal cells capable of conforming to the hemispherical image plane of a miniature ball lens. In addition, we have shown the imaging capability of this device. Optical testing shows that the fabrication process that allows hemispherical coverage does not compromise the ability to form functional photodetectors. It remains to develop a process for fabricating a hemispherical CMOS imager based on arrays of interconnected hexagonal cells.

\section{ACKNOWLEDGEMENTS}

A patent disclosure has been filed. We acknowledge the use of the Stanford Nanofabrication Facility (SNF), a node of the National Nanofabrication Infrastructure Network (NNIN), and the Stanford Nano Shared Facilities (SNSF) for sample fabrication and characterization. The authors acknowledge Drs Sean Stetson and David Frakes of Google ATAP for initiating and funding this project. We also thank Xin Lei and Dr Kimberly Harrison of Stanford for valuable discussion.

\section{COMPETING INTERESTS}

The authors declare no conflict of interest.

\section{REFERENCES}

1 Ford J, Stamenov I, Olivas SJ et al. Fiber-coupled monocentric lens imaging. Computational Optical Sensing and Imaging 2013; 23-27 Jun 2013; Arlington, Virginia, USA; 2013: CW4C.2.

2 Tremblay EJ, Marks DL, Brady DJ et al. Design and scaling of monocentric multiscale imagers. Applied Optics 2012; 51: 4691-4702.

3 Stamenov I, Arianpour A, Olivas SJ et al. Panoramic monocentric imaging using fiber-coupled focal planes. Optics Express 2014; 22: 31708-31721.

4 Itonaga K, Arimura T, Matsumoto K et al. A novel curved CMOS image sensor integrated with imaging system. 2014 Symposium on VLSI Technology: Digest of Technical Papers; 9-12 Jun 2014; Honolulu, HI, USA; 2014: 1-2.

5 Song YM, Xie Y, Malyarchuk $V$ et al. Digital cameras with designs inspired by the arthropod eye. Nature 2013; 497: 95-99.

$6 \mathrm{Ko} \mathrm{HC}$, Stoykovich MP, Song J et al. A hemispherical electronic eye camera based on compressible silicon optoelectronics. Nature 2008; 454: 748-753.

7 Lü C, Li M, Xiao J et al. Mechanics of tunable hemispherical electronic eye camera systems that combine rigid device elements with soft elastomers. Journal of Applied Mechanics 2013; 80: 061022.

8 Huang CC, Wu X, Liu H et al. Large-field-of-view wide-spectrum artificial reflecting superposition compound eyes. Small 2014; 10: 3050-3057.

9 Dinyari R, Rim S-B, Huang $\mathrm{K}$ et al. Curving monolithic silicon for nonplanar focal plane array applications. Applied Physics Letters 2008; 92: 091114.

10 Dinyari R, Loudin JD, Huie P et al. A curvable silicon retinal implant. 2009 IEEE International Electron Devices Meeting (IEDM); 7-9 Dec 2009; Baltimore, MD, USA; 2009: 1-4.

11 Wang L, Mathieson K, Kamins TI et al. Photovoltaic retinal prosthesis: Implant fabrication and performance. Journal of Neural Engineering 2012; 9: 046014.

12 Mathieson K, Loudin J, Goetz G et al. Photovoltaic retinal prosthesis with high pixel density. Nature Photonics 2012; 6: 872-872.

13 Liu C-Y, Yang F, Teng C-C et al. A contact-lens-shaped IC chip technology. Journal of Micromechanics and Microengineering 2014; 24: 045025.

14 Hsieh CY, Chen CS, Tsou WA et al. A flexible mixed-signal/RF CMOS technology for implantable electronics applications. Journal of Micromechanics and Microengineering 2010; 20: 045017.

15 Shahrjerdi D, Bedell SW. Extremely flexible nanoscale ultrathin body silicon integrated circuits on plastic. Nano Letters 2013; 13: 315-320.

16 The Math Forum @ Drexel. Making Hemispheres out of Paper. Available at http:// mathforum.org/library/drmath/view/55168.html, accessed on 14 March 2016.

17 Fossum ER. CMOS image sensors: electronic camera-on-a-chip. IEEE Transactions on Electron Devices 1997; 44: 1689-1698.

18 Yuko U, Tanemasa A. CMOS image sensor using SOI-MOS/photodiode composite photodetector device. Japanese Journal of Applied Physics 2002; 41: 2620.

19 Borrelli NF, Brady MD, Burt RL et al. Image sensor using thin-film SOI. US Patent Application US20080070340, 2008.

20 Hoffman A, Loose M, Suntharalingam V. CMOS detector technology. Experimental Astronomy 2006; 19: 111-134.

21 Fossum ER, Hondongwa DB. A review of the pinned photodiode for CCD and CMOS image sensors. IEEE Journal of the Electron Devices Society 2014; 2: 33-43.

22 Baltes $\mathrm{H}$, Brand O, Fedder GK et al. CMOS-MEMS: Advanced Micro and Nanosystems. John Wiley \& Sons: New York City, NY, United States. 2005.

23 Palik ED. Handbook of Optical Constants of Solids. Elsevier Science: Amsterdam, the Netherlands. 2012.

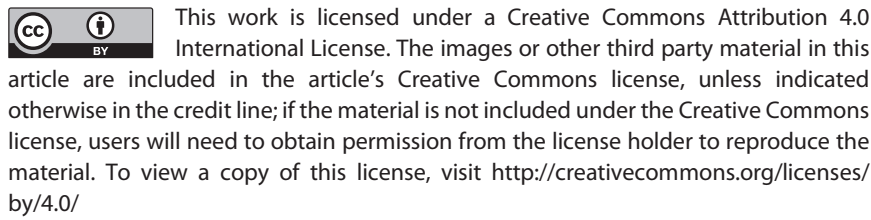

This work is licensed under a Creative Commons Attribution 4.0 nternational License. The images or other third party material in this license, users will need to obtain permission from the license holder to reproduce the by/4.0/

Supplementary Information for this article can be found on the Microsystems \& Nanoengineering website: http://www.nature.com/ micronano. 